\title{
Less Conservative Optimal Robust Control of a 3-DOF Helicopter
}

\author{
L. F. S. Buzachero, ${ }^{1}$ E. Assunção, ${ }^{2}$ M. C. M. Teixeira, ${ }^{2}$ and E. R. P. da Silva ${ }^{3}$ \\ ${ }^{1}$ Universidade Tecnológica Federal do Paraná (UTFPR), 86812-460 Apucarana, PR, Brazil \\ ${ }^{2}$ Research Laboratory in Control, Department of Electrical Engineering, Universidade Estadual Paulista (UNESP), \\ Campus of Ilha Solteira, 15385-000 Ilha Solteira, SP, Brazil \\ ${ }^{3}$ Universidade Tecnológica Federal do Paraná (UTFPR), 86300-000 Cornélio Procópio, PR, Brazil
}

Correspondence should be addressed to L. F. S. Buzachero; luiz.buzachero@yahoo.com.br

Received 6 October 2014; Accepted 14 January 2015

Academic Editor: Yongji Wang

Copyright (C) 2015 L. F. S. Buzachero et al. This is an open access article distributed under the Creative Commons Attribution License, which permits unrestricted use, distribution, and reproduction in any medium, provided the original work is properly cited.

\begin{abstract}
This work proposes an improved technique for design and optimization of robust controllers norm for uncertain linear systems, with state feedback, including the possibility of time-varying the uncertainty. The synthesis techniques used are based on LMIs (linear matrix inequalities) formulated on the basis of Lyapunov's stability theory, using Finsler's lemma. The design has used the addition of the decay rate restriction, including a parameter $\gamma$ in the LMIs, responsible for decreasing the settling time of the feedback system. Qualitative and quantitative comparisons were made between methods of synthesis and optimization of the robust controllers norm, seeking alternatives with lower cost and better performance that meet the design restrictions. A practical application illustrates the efficiency of the proposed method with a failure purposely inserted in the system.
\end{abstract}

\section{Introduction}

The history of linear matrix inequalities (LMIs) in the analysis of dynamical systems dates back to over 100 years. The story begins around 1890 when Lyapunov published his work introducing what is now called Lyapunov's theory [1]. The researches and publications involving Lyapunov's theory have grown up a lot in recent decades [2], opening a very wide range for various approaches such as robust stability analysis of linear systems [3].

In addition to the various current controllers design techniques, the design of optimal robust controllers (or controller design by quadratic stability) using LMI stands out for solving problems that previously had no known solution [4]. These designs use specialized computer packages [5].

Recent publications have found a certain conservatism inserted in the analysis of quadratic stability, which led to a search for solutions to eliminate this conservatism. Finsler's lemma [6] has been widely used in control theory for the stability analysis by LMIs, with results similar to the LMI quadratic stability, but with extra matrices, allowing a certain relaxation in the stability analysis (referred as "extended" stability), by obtaining a larger region of feasibility. However, these methods only guarantee stability for uncertain timeinvariant systems or with very small variation rate [7].

The main focus of this paper is to propose an improved method of design and optimization technique than the one presented in [8], including the possibility of time-varying the uncertainty, searching for lower gains of controllers that have the same dynamic performance when the system is forced to have a faster transient with the inclusion of decay rate restriction (a $\mathscr{D}$-stability performance index [9] which ensures that the eigenvalues of the uncertain system are on the left of $\gamma$ scalar) in the LMIs formulation.

Comparisons will be made through a practical application in a Quanser's 3-DOF helicopter [10], considering a failure during the landing treated as a time variant uncertainty, and a generic analysis involving 1000 randomly generated polytopic uncertain systems. 
The notation used throughout the paper is standard. The symbol $\left({ }^{\prime}\right)$ indicates transpose and $\left({ }^{-1}\right)$ indicates the inverse matrix; $P>0$ means that $P$ is symmetric positive definite. $\mathbb{N}$ denotes the nonnegative integers $\{0,1,2, \ldots\}$ and $\mathbb{R}$ the real numbers.

\section{Robust Stability Using Decay Rate as a Performance Index}

Consider the controllable uncertain linear time-invariant system described in the state space form:

$$
\dot{x}(t)=A(\alpha) x(t)+B(\alpha) u(t) .
$$

This system can be described as convex combination of the polytope vertexes:

$$
\dot{x}(t)=\sum_{j=1}^{r} \alpha_{j} A_{j} x(t)+\sum_{j=1}^{r} \alpha_{j} B_{j} u(t)
$$

with

$$
\alpha_{j}>0, \quad j=1, \ldots, r, \quad \sum_{j=1}^{r} \alpha_{j}=1,
$$

where $r$ is the number of the polytope vertexes [1].

Considering the uncertain system (2) and exiting Lyapunov theory for designing controllers, the following theorem is stated [1].

Theorem 1. A sufficient condition for the uncertain system (2) stability guarantee subject to decay rate greater than or equal to $\gamma$ is the existence of matrices $X=X^{\prime} \in \mathbb{R}^{n \times n}$ and $G \in \mathbb{R}^{m \times n}$, such that

$$
\begin{gathered}
A_{j} X-B_{j} G+X A_{j}^{\prime}-G^{\prime} B_{j}^{\prime}+2 \gamma X<0, \\
X>0
\end{gathered}
$$

with $j=1, \ldots, r$.

When the LMIs (4) and (5) are feasible, a state feedback matrix that stabilizes the system can be found as

$$
K=G X^{-1} \text {. }
$$

Proof. See [1].

Thus, the feedback of the uncertain system presented in (1) can be done, with (4) and (5) being sufficient conditions for asymptotic stability of the polytope for a state feedback system with decay rate restriction. If the solution of LMIs is feasible, the uncertain system's stability is guaranteed.

In many situations the norm of the state feedback matrix is high, precluding its practical application. In [11] an optimization method that minimizes the gains of the controller designed via LMIs (4) and (5) was proposed, but this does not eliminate the inherent conservatism.

Thus, in [8] a new way to optimize the norm of $K$ controller was presented eliminating the conservatism of the LMIs by Finsler's lemma.
We can use the Finsler's lemma to express the stability in terms of LMIs, with advantages over the existing Lyapunov's theory [1], once it introduces new variables $(\mu, \mathscr{X})$ under conditions which involve only $\mathscr{L}, \mathscr{B}$, and $\mathscr{B}^{\perp}$.

Lemma 2 (Finsler). Consider $w \in \mathbb{R}^{n_{x}}, \mathscr{L} \in \mathbb{R}^{n_{x} \times n_{x}}$, and $\mathscr{B} \in \mathbb{R}^{m_{x} \times n_{x}}$ with $\operatorname{rank}(\mathscr{B})<n_{x}$ and $\mathscr{B}^{\perp}$ a basis for the null space of $\mathscr{B}$ (i.e., $\mathscr{B} \mathscr{B}^{\perp}=0$ ). Then the following conditions are equivalent:

$$
\begin{aligned}
& \text { (1) } w^{\prime} \mathscr{L} w<0, \forall w \neq 0: \mathscr{B} w=0 \\
& \text { (2) } \mathscr{B}^{\perp^{\prime}} \mathscr{L} \mathscr{B}^{\perp}<0 \\
& \text { (3) } \exists \mu \in \mathbb{R}: \mathscr{L}-\mu \mathscr{B}^{\prime} \mathscr{B}<0 \\
& \text { (4) } \exists \mathscr{X} \in \mathbb{R}^{n_{x} \times m_{x}}: \mathscr{L}+\mathscr{X} \mathscr{B}+\mathscr{B}^{\prime} \mathscr{X}^{\prime}<0 .
\end{aligned}
$$

Proof. See [6] or [12].

2.1. Robust Stability of Systems Using Finsler's Lemma with Decay Rate Restriction. Defining $w=\left[\begin{array}{l}x \\ \dot{x}\end{array}\right], \mathscr{B}=$ $[(A-B K)-I], \mathscr{B}^{\perp}=\left[\begin{array}{c}I \\ (A-B K)\end{array}\right]$, and $\mathscr{L}=\left[\begin{array}{cc}2 \gamma P & P \\ P & 0\end{array}\right]$, note that $\mathscr{B} w=0$ corresponds to the feedback system with $K$ and $w^{\prime} \mathscr{L} w<0$ represents the stability restriction with decay rate formulated from the quadratic Lyapunov function [1]. In this case the dimensions of Lemma's 2 variables are $n_{x}=2 n$ and $m_{x}=n$.

Thus, it is possible to characterize stability through the quadratic Lyapunov function $\left(V(x(t)):=x(t)^{\prime} P x(t)\right)$, generating new degrees of freedom for the synthesis of controllers.

From existing proof of Finsler's lemma it can be concluded that the properties of one to four are equivalent. Thus, we can rewrite fourth property as follows:

(4) $\exists \mathscr{X} \in \mathbb{R}^{2 n \times n}, P=P^{\prime}>0$ such that

$$
\left[\begin{array}{cc}
2 \gamma P & P \\
P & 0
\end{array}\right]+\mathscr{X}[(A-B K)-I]+\left[\begin{array}{c}
(A-B K)^{\prime} \\
-I
\end{array}\right] X^{\prime}<0
$$

conveniently choosing the matrix variables $\mathscr{X}=\left[\begin{array}{c}Z \\ a Z\end{array}\right]$, with $Z \in \mathbb{R}^{n \times n}$ nonsymmetric and $a$ a relaxation constant that has the function of flexible matrix $\mathscr{X}$ in the LMI [13]. This constant can be defined by making a one-dimensional search. Applying the congruence transformation $\left[\begin{array}{cc}Z^{-1} & 0 \\ 0 & Z^{-1}\end{array}\right]$ at the left and $\left[\begin{array}{cc}Z^{-1} & 0 \\ 0 & Z^{-1}\end{array}\right]^{\prime}$ at the right, in the fourth property, and making $Y=\left(Z^{\prime}\right)^{-1}, G=K Y$, and $Q=Y^{\prime} P Y$, the following LMIs were found:

$$
\begin{gathered}
{\left[\begin{array}{cc}
A Y+Y^{\prime} A^{\prime}-B G-G^{\prime} B^{\prime}+2 \gamma Q & Q+a Y^{\prime} A^{\prime}-a G^{\prime} B^{\prime}-Y \\
Q+a A Y-a B G-Y^{\prime} & -a Y-a Y^{\prime}
\end{array}\right]} \\
\qquad \begin{array}{c}
<>0 \\
\multicolumn{2}{c}{}
\end{array}
\end{gathered}
$$

with $Y \in \mathbb{R}^{n \times n}, Y \neq Y^{\prime}, G \in \mathbb{R}^{m \times n}$, and $Q \in \mathbb{R}^{n \times n}$.

These LMIs meet the restrictions for the asymptotic stability of the system with state feedback. The stability resulting of the LMIs derived from Finsler's lemma referred 
to as extended stability [14]. The advantage of using Finsler's lemma formulation for robust stability analysis is the freedom of Lyapunov's function, now defined as $Q(\alpha)=\sum_{j=1}^{r} \alpha_{j} Q_{j}$, $\sum_{j=1}^{r} \alpha_{j}=1, \alpha_{j} \geq 0$ and $j=1, \ldots, r$. As $Q(\alpha)$ depends on $\alpha$, the Lyapunov matrix use fits to time-invariant polytopic uncertainties, with permitted rate of variation being sufficiently small. Thus in [8] the following theorem was presented.

Theorem 3. In order to guarantee the stability of the uncertain system (2) subject to decay rate greater than or equal to $\gamma$, a sufficient condition is the existence of matrices $Y \in \mathbb{R}^{n \times n}, Q_{j}=$ $Q_{j}^{\prime} \in \mathbb{R}^{n \times n}$, and $G \in \mathbb{R}^{m \times n}$, such that

$$
\left[\begin{array}{cc}
A_{j} Y+Y^{\prime} A_{j}^{\prime}-B_{j} G-G^{\prime} B_{j}^{\prime}+2 \gamma Q_{j} & Q_{j}+a Y^{\prime} A_{j}^{\prime}-a G^{\prime} B_{j}^{\prime}-Y \\
Q_{j}+a A_{j} Y-a B_{j} G-Y^{\prime} & -a Y-a Y^{\prime}
\end{array}\right]<0,
$$

$$
Q_{j}>0
$$

with $j=1, \ldots, r$.

When LMIs (9) and (10) are feasible, a state feedback matrix that stabilizes the system can be given by

$$
K=G Y^{-1} \text {. }
$$

Proof. See [8].

Thus, it can be feedback into the uncertain system with (9) and (10) sufficient conditions for asymptotic stability of the polytope.

2.2. Optimization of $K$ Norm Using Finsler's Lemma. In [8] there was a difficulty in applying the existing theory for the optimization of $K$ matrix norm [11] for the new structure of LMIs. This was due to the nonsymmetry of $Y$ matrix for the controller synthesis, condition that was necessary to the LMI development when the controller synthesis matrix was $X=P^{-1}$. The solution found was using the idea of the optimization procedure for redesign presented in [15]. Thus, in [8] the new optimization method adequacy was proposed, with the minimization of a scalar $\beta$ obeying the relation $K^{\prime} K<\beta P_{j}$ with $P_{j}$ the Lyapunov function, to the new relaxed parameters through the following theorem.

Theorem 4. A constraint for the $K \in \mathbb{R}^{m \times n}$ matrix norm of state feedback can be obtained, with $K=G Y^{-1}$ and $Q_{j}=$ $Y^{\prime} P_{j} Y$, being $Y \in \mathbb{R}^{n \times n}, G \in \mathbb{R}^{m \times n}$, and $P_{j} \in \mathbb{R}^{n \times n}, P_{j}=$ $P_{j}^{\prime}>0$ finding the minimum $\beta, \beta>0$, such that $K^{\prime} K<\beta P_{j}$, $j=1, \ldots, N$. One can get the optimal value of $\beta$ solving the optimization problem with the LMIs:

$$
\begin{array}{ll}
\min & \beta \\
\text { s.t. } & {\left[\begin{array}{cc}
Q_{j} & G^{\prime} \\
G & \beta I_{m}
\end{array}\right]>0}
\end{array}
$$

(LMIs given in (9)), where $I_{m}$ denotes the identity matrix of $m$ order.

Proof. See [8].
This way of optimizing the norm of $K$ showed better results than the one presented in [11]. However, the optimization LMI, in order to be bound by the Lyapunov's matrix $P_{j}$, still does not have the minimal gains that would be found to meet the design requirements.

In order to improve the optimization performance, an alternative is presented in Section 2.4 to optimize the norm of $K$ controller reducing the conservatism of the LMIs design with a convenient manipulation.

2.3. Robust Stability of Systems Using Reciprocal Projection Lemma with the Decay Rate Restriction. In order to verify the advantages of the new formulation proposed in Section 2.4, one of the best examples of optimal design of $K$ was borrowed from [8] for comparison purposes. As in the extended stability case, the advantage of using the reciprocal projection lemma for robust stability analysis is Lyapunov's function degree of freedom, now defined as $P(\alpha)=\sum_{j=1}^{r} \alpha_{j} P_{j}$, $\sum_{j=1}^{r} \alpha_{j}=1, \alpha_{j} \geq 0$ and $j=1, \ldots, r$. As described before Theorem 3, the use of $P(\alpha)$ fits to time-invariant polytopic uncertainties, with permitted rate of variation being sufficiently small. To verify this, Theorem 5 follows.

Theorem 5. A sufficient condition which guarantees the stability of the uncertain system (2) is the existence of matrices $V \in \mathbb{R}^{n \times n}, P_{j}=P_{j}^{\prime} \in \mathbb{R}^{n \times n}$, and $Z \in \mathbb{R}^{m \times n}$, such that LMIs (13) are met as follows:

$$
\begin{aligned}
& {\left[\begin{array}{ccc}
-\left(V+V^{\prime}\right) & V^{\prime} A_{j}^{\prime}-Z^{\prime} B_{j}^{\prime}+\gamma V^{\prime}+P_{j} & V^{\prime} \\
A_{j} V-B_{j} Z+\gamma V+P_{j} & -P_{j} & 0 \\
V & 0 & -P_{j}
\end{array}\right]} \\
& <0, \\
& P_{j}>0
\end{aligned}
$$

with $j=1, \ldots, r$.

When the LMIs (13) are feasible, a state feedback matrix which stabilizes the system can be given by

$$
K=Z V^{-1} \text {. }
$$

Proof. See [8].

Theorem 6 shows the optimization of $K$ for LMIs (13).

Theorem 6. A constraint for the $K \in \mathbb{R}^{m \times n}$ matrix norm of state feedback is obtained, with $K=Z V^{-1}, V \in \mathbb{R}^{n \times n}$, and $Z \in$ $\mathbb{R}^{m \times n}$ finding the minimum $\beta, \beta>0$, such that $K^{\prime} K<\beta M$, being $M=V^{\prime-1} V^{-1}$ and therefore $M=M^{\prime}>0$. We can get the optimal value of $\beta$ solving the optimization problem with the LMIs as follows:

$$
\begin{array}{ll}
\min & \beta \\
\text { s.t. } & {\left[\begin{array}{cc}
I_{n} & Z^{\prime} \\
Z & \beta I_{m}
\end{array}\right]>0}
\end{array}
$$


where $I_{m}$ and $I_{n}$ denote the identity matrices of $m$ and $n$ order, respectively.

Proof. See [8].

2.4. New Formulation for Robust Stability of Systems Using Finsler's Lemma with Decay Rate Restriction. Defining $\mathscr{B}=\left[\begin{array}{ll}A^{\prime} & -I\end{array}\right], \mathscr{B}^{\perp}=\left[\begin{array}{c}I \\ A^{\prime}\end{array}\right]$ and also consider $\mathscr{L}=\left[\begin{array}{cc}-B G-G^{\prime} B^{\prime}+2 \gamma X & X \\ X & 0\end{array}\right]$. Check that the lemma variables (1) dimensions are $n_{x}=2 n$ and $m_{x}=n$. Considering that $X$ is the matrix used to define the quadratic Lyapunov function, we will have the second propriety of Finsler's lemma written as follows:

(2) $\exists X=X^{\prime}>0$ such that

$$
\left[\begin{array}{c}
I \\
A^{\prime}
\end{array}\right]^{\prime}\left[\begin{array}{cc}
-B G-G^{\prime} B^{\prime}+2 \gamma X & X \\
X & 0
\end{array}\right]\left[\begin{array}{c}
I \\
A^{\prime}
\end{array}\right]<0
$$

which results in the necessary and sufficient condition for the system's stabilizability, including decay rate:

(2) $A X-B G+X A^{\prime}-G^{\prime} B^{\prime}+2 \gamma X<0$.

Thus, it is possible to characterize stability through the quadratic Lyapunov function $\left(V(x(t))=x(t)^{\prime} P x(t)\right)$, generating further degrees of freedom for the controllers synthesis.

From existing Finsler's lemma proof (Lemma 2), it can be concluded that the second and fourth properties are equivalent. Thus, we can rewrite the fourth property as follows:
(4) $\exists \mathscr{Y} \in \mathbb{R}^{2 n \times n}, X=X^{\prime}>0$ such that

$$
\left[\begin{array}{cc}
-B G-G^{\prime} B^{\prime}+2 \gamma X & X \\
X & 0
\end{array}\right]+\mathscr{Y}\left[\begin{array}{ll}
A^{\prime} & -I
\end{array}\right]+\left[\begin{array}{c}
A \\
-I
\end{array}\right] \mathscr{Y}^{\prime}<0 .
$$

Conveniently choose the matrix variables $\mathscr{Y}=\left[\begin{array}{l}Y_{1} \\ Y_{2}\end{array}\right]$, with $Y_{1}, Y_{2} \in \mathbb{R}^{n \times n}$. Developing the fourth property, we have

$$
\begin{gathered}
{\left[\begin{array}{cc}
-B G-G^{\prime} B^{\prime}+2 \gamma X & X \\
X & 0
\end{array}\right]+\left[\begin{array}{ll}
Y_{1} A^{\prime} & -Y_{1} \\
Y_{2} A^{\prime} & -Y_{2}
\end{array}\right]} \\
+\left[\begin{array}{cc}
A Y_{1}^{\prime} & A Y_{2}^{\prime} \\
-Y_{1}^{\prime} & -Y_{2}^{\prime}
\end{array}\right]<0 .
\end{gathered}
$$

Thus the following LMIs subject to decay rate greater than or equal to $\gamma$ were found:

$$
\left[\begin{array}{cc}
A Y_{1}^{\prime}-B G+Y_{1} A^{\prime}-G^{\prime} B^{\prime}+2 \gamma X & X-Y_{1}+A Y_{2}^{\prime} \\
X-Y_{1}^{\prime}+Y_{2} A^{\prime} & -Y_{2}-Y_{2}^{\prime}
\end{array}\right]<0,
$$

$$
X>0
$$

with $Y_{1}$ and $Y_{2} \in \mathbb{R}^{n \times n}, Y_{1} \neq Y_{1}^{\prime}$ and $Y_{2} \neq Y_{2}^{\prime}, G \in \mathbb{R}^{m \times n}$, and $X \in \mathbb{R}^{n \times n}, X=X^{\prime}>0$.

These LMIs meet the restrictions for the asymptotic stability of the system with state feedback. It can be seen that the first principal minor of LMI (19) has the structure of the results found with the theorem of stability with decay rate. However, there is also, as stated in Finsler's lemma, a high degree of freedom, due to the relaxation variable matrices $Y_{1}$ and $Y_{2}$, without being symmetric, and for a robust stability approach, they may be polytopic: $Y_{1}(\alpha)=\sum_{j=1}^{r} \alpha_{j} Y_{1 j}$ and $Y_{2}(\alpha)=\sum_{j=1}^{r} \alpha_{j} Y_{2 j}, \sum_{j=1}^{r} \alpha_{j}=1, \alpha_{j} \geq 0$ and $j=1, \ldots, r$. Therefore the following theorem is proposed.

Theorem 7. In order to guarantee the stability of the uncertain system (2) subject to decay rate greater than or equal to $\gamma$ a sufficient condition is the existence of matrices $Y_{1 j}$ and $Y_{2 j} \in$ $\mathbb{R}^{n \times n}, X=X^{\prime} \in \mathbb{R}^{n \times n}$, and $G \in \mathbb{R}^{m \times n}$, such that

$$
\begin{gathered}
{\left[\begin{array}{cc}
A_{j} Y_{1 j}^{\prime}-B_{j} G+Y_{1 j} A_{j}^{\prime}-G^{\prime} B_{j}^{\prime}+2 \gamma X & X-Y_{1 j}+A_{j} Y_{2 j}^{\prime} \\
X-Y_{1 j}^{\prime}+Y_{2 j} A_{j}^{\prime} & -Y_{2 j}-Y_{2 j}^{\prime}
\end{array}\right]<0} \\
j=1, \ldots, r, \\
{\left[\begin{array}{cc}
A_{j} Y_{1 k}^{\prime}-B_{j} G+A_{k} Y_{1 j}^{\prime}-B_{k} G+Y_{1 k} A_{j}^{\prime}-G^{\prime} B_{j}^{\prime}+Y_{1 j} A_{k}^{\prime}-G^{\prime} B_{k}^{\prime}+4 \gamma X & 2 X-Y_{1 j}-Y_{1 k}+A_{k} Y_{2 j}^{\prime}+A_{j} Y_{2 k}^{\prime} \\
2 X-Y_{1 j}^{\prime}-Y_{1 k}^{\prime}+Y_{2 j} A_{k}^{\prime}+Y_{2 k} A_{j}^{\prime} & -Y_{2 j}-Y_{2 j}^{\prime}-Y_{2 k}-Y_{2 k}^{\prime}
\end{array}\right]<0} \\
j=1, \ldots, r-1 ; \quad k=j+1, \ldots, r,
\end{gathered}
$$


When LMIs (21), (22), and (23) are feasible, a state feedback matrix that stabilizes the system can be given by

$$
K=G X^{-1} .
$$

$$
\begin{aligned}
\sum_{j=1}^{r} \alpha_{j}^{2} & {\left[\begin{array}{cc}
A_{j} Y_{1 j}^{\prime}-B_{j} G+Y_{1 j} A_{j}^{\prime}-G^{\prime} B_{j}^{\prime}+2 \gamma X & X-Y_{1 j}+A_{j} Y_{2 j}^{\prime} \\
X-Y_{1 j}^{\prime}+Y_{2 j} A_{j}^{\prime} & -Y_{2 j}-Y_{2 j}^{\prime}
\end{array}\right] } \\
& +\sum_{j=1}^{r-1} \sum_{k=j+1}^{r} \alpha_{j} \alpha_{k}\left[\begin{array}{cc}
A_{j} Y_{1 k}^{\prime}-B_{j} G+A_{k} Y_{1 j}^{\prime}-B_{k} G+Y_{1 k} A_{j}^{\prime}-G^{\prime} B_{j}^{\prime}+Y_{1 j} A_{k}^{\prime}-G^{\prime} B_{k}^{\prime}+4 \gamma X & 2 X-Y_{1 j}-Y_{1 k}+A_{k} Y_{2 j}^{\prime}+A_{j} Y_{2 k}^{\prime} \\
2 X-Y_{1 j}^{\prime}-Y_{1 k}^{\prime}+Y_{2 j} A_{k}^{\prime}+Y_{2 k} A_{j}^{\prime} & -Y_{2 j}-Y_{2 j}^{\prime}-Y_{2 k}-Y_{2 k}^{\prime}
\end{array}\right]
\end{aligned}
$$$$
<0 \text {. }
$$

Knowing that generically

$$
\begin{aligned}
\sum_{i=1}^{r} \alpha_{i} \sum_{j=1}^{r} \alpha_{j} & =\sum_{j=1}^{r} \alpha_{j}^{2}+2 \sum_{j=1}^{r-1} \sum_{k=j+1}^{r} \alpha_{j} \alpha_{k}, \\
\sum_{i=1}^{r} \alpha_{i} \sum_{j=1}^{r} \alpha_{j} H_{i} R_{j}= & \sum_{j=1}^{r} \alpha_{j}^{2} H_{j} R_{j} \\
& +\sum_{j=1}^{r-1} \sum_{k=j+1}^{r} \alpha_{j} \alpha_{k}\left(H_{j} R_{k}+H_{k} R_{j}\right)
\end{aligned}
$$

thus the following equivalences are true:

$$
\begin{aligned}
\sum_{i=1}^{r} \alpha_{i} \sum_{j=1}^{r} \alpha_{j} A_{i} Y_{1 j}^{\prime}= & \sum_{j=1}^{r} \alpha_{j}^{2} A_{j} Y_{1 j}^{\prime} \\
& +\sum_{j=1}^{r-1} \sum_{k=j+1}^{r} \alpha_{j} \alpha_{k}\left(A_{j} Y_{1 k}^{\prime}+A_{k} Y_{1 j}^{\prime}\right),
\end{aligned}
$$

Proof. Assume that LMIs (21), (22), and (23) are feasible. Considering $\alpha_{j}>0$ for $j=1, \ldots, r$, then we have

$$
\begin{gathered}
\sum_{i=1}^{r} \alpha_{i} \sum_{j=1}^{r} \alpha_{j} B_{j} G=\sum_{j=1}^{r} \alpha_{j}^{2} B_{j} G+\sum_{j=1}^{r-1} \sum_{k=j+1}^{r} \alpha_{j} \alpha_{k}\left(B_{j} G+B_{k} G\right) \\
\sum_{i=1}^{r} \alpha_{i} \sum_{j=1}^{r} \alpha_{j} Y_{1 j}=\sum_{j=1}^{r} \alpha_{j}^{2} Y_{1 j}+\sum_{j=1}^{r-1} \sum_{k=j+1}^{r} \alpha_{j} \alpha_{k}\left(Y_{1 j}+Y_{1 k}\right) \\
\sum_{i=1}^{r} \alpha_{i} \sum_{j=1}^{r} \alpha_{j} Y_{2 j}=\sum_{j=1}^{r} \alpha_{j}^{2} Y_{2 j}+\sum_{j=1}^{r-1} \sum_{k=j+1}^{r} \alpha_{j} \alpha_{k}\left(Y_{2 j}+Y_{2 k}\right) \\
\sum_{i=1}^{r} \alpha_{i} \sum_{j=1}^{r} \alpha_{j} X=\sum_{j=1}^{r} \alpha_{j}^{2} X+2 \sum_{j=1}^{r-1} \sum_{k=j+1}^{r} \alpha_{j} \alpha_{k} X
\end{gathered}
$$

So, (25) can be rewritten as

$$
\sum_{i=1}^{r} \alpha_{i} \sum_{j=1}^{r} \alpha_{j}\left[\begin{array}{cc}
A_{i} Y_{1 j}^{\prime}-B_{j} G+Y_{1 i} A_{j}^{\prime}-G^{\prime} B_{j}^{\prime}+2 \gamma X & X-Y_{1 j}+A_{i} Y_{2 j}^{\prime} \\
X-Y_{1 j}^{\prime}+Y_{2 i} A_{j}^{\prime} & -Y_{2 j}-Y_{2 j}^{\prime}
\end{array}\right]<0
$$

and consequently

$$
\left[\begin{array}{cc}
\sum_{i=1}^{r} \alpha_{i} A_{i} \sum_{j=1}^{r} \alpha_{j} Y_{1 j}^{\prime}-\sum_{j=1}^{r} \alpha_{j} B_{j} G+\sum_{i=1}^{r} \alpha_{i} Y_{1 i} \sum_{j=1}^{r} \alpha_{j} A_{j}^{\prime}-G^{\prime} \sum_{j=1}^{r} \alpha_{j} B_{j}^{\prime}+2 \gamma X & X-\sum_{j=1}^{r} \alpha_{j} Y_{1 j}+\sum_{i=1}^{r} \alpha_{i} A_{i} \sum_{j=1}^{r} \alpha_{j} Y_{2 j}^{\prime} \\
X-\sum_{j=1}^{r} \alpha_{j} Y_{1 j}^{\prime}+\sum_{i=1}^{r} \alpha_{i} Y_{2 i} \sum_{j=1}^{r} \alpha_{j} A_{j}^{\prime} & -\sum_{j=1}^{r} \alpha_{j} Y_{2 j}-\sum_{j=1}^{r} \alpha_{j} Y_{2 j}^{\prime}
\end{array}\right]<0 .
$$

Thus (29) can be rewritten as

$$
\left[\begin{array}{cc}
A(\alpha) Y_{1}^{\prime}(\alpha)-B(\alpha) G+Y_{1}(\alpha) A^{\prime}(\alpha)-G^{\prime} B^{\prime}(\alpha)+2 \gamma X & X-Y_{1}(\alpha)+A(\alpha) Y_{2}^{\prime}(\alpha) \\
X-Y_{1}^{\prime}(\alpha)+Y_{2}(\alpha) A^{\prime}(\alpha) & -Y_{2}(\alpha)-Y_{2}^{\prime}(\alpha)
\end{array}\right]<0
$$


where $Y_{1}(\alpha)=\sum_{j=1}^{r} \alpha_{j} Y_{1 j}$ and $Y_{2}(\alpha)=\sum_{j=1}^{r} \alpha_{j} Y_{2 j}$, with $\sum_{j=1}^{r} \alpha_{j}=1, \alpha_{j} \geq 0$ and $j=1, \ldots, r$.

There is a great advantage in using the new formulations (21) and (22) using Finsler's lemma compared with the old one (9) due to insertion of two polytopic matrices $Y_{1 j}$ and $Y_{2 j}$, relaxing more the LMIs when compared to the old formulation where there was only the polytopic Lyapunov matrix $Q_{i}$; moreover as described before Theorem 3, the use of $Q(\alpha)$ fits to time-invariant polytopic uncertainties, with permitted rate of variation being sufficiently small, unlike this new formulation where the Lyapunov matrix $X$ is not polytopic, allowing variations in $\alpha$.

Below we have an alternative optimization of the norm of $K$, which together with LMIs (21), (22), and (23) shows better results for the controller gains, as will be seen in Section 4 .

Theorem 8. Given a constant $\mu_{0}>0$, a constraint for the state feedback $K \in \mathbb{R}^{m \times n}$ matrix norm is obtained, with $K=G X^{-1}$, $X=X^{\prime}>0, X \in \mathbb{R}^{n \times n}$, and $G \in \mathbb{R}^{m \times n}$ by finding the minimum of $\beta, \beta>0$ such that $K^{\prime} K<\left(\beta / \mu_{0}\right) I_{n}$. We can get the minimum $\beta$ solving the follwoing optimization problem:

$$
\begin{gathered}
\min \quad \beta \\
\text { s.t. } \quad\left[\begin{array}{cc}
X & G^{\prime} \\
G & \beta I_{m}
\end{array}\right]>0 \\
X>\mu_{0} I_{n} \\
(\text { LMIs (18) or (20) and (21)), }
\end{gathered}
$$

where $I_{m}$ and $I_{n}$ denote identity matrices of $m$ and $n$ order, respectively.

Proof. Applying the Schur complement for the first inequality of (31) results in

$$
\begin{gathered}
\beta I_{m}>0, \\
X-G^{\prime}\left(\beta I_{m}\right)^{-1} G>0 .
\end{gathered}
$$

Thus, from (35) we obtain

$$
X>\frac{1}{\beta} G^{\prime} G \Longrightarrow G^{\prime} G<\beta X .
$$

Replacing $G=K X$ in (36) results in

$$
X K^{\prime} K X<\beta X \Longrightarrow K^{\prime} K<\beta X^{-1} .
$$

From (32) we obtain

$$
X^{-1}<\frac{1}{\mu_{0}} I_{n}
$$

So from (37) and (38),

$$
K^{\prime} K<\frac{\beta}{\mu_{0}} I_{n}
$$

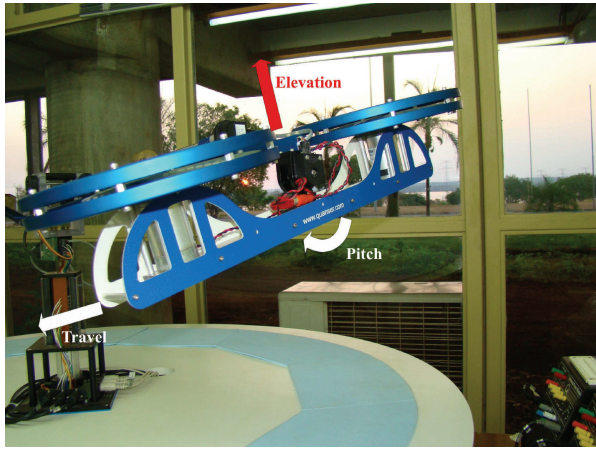

FIGURE 1: Quanser's 3-DOF helicopter of Control Research Laboratory at FEIS, UNESP.

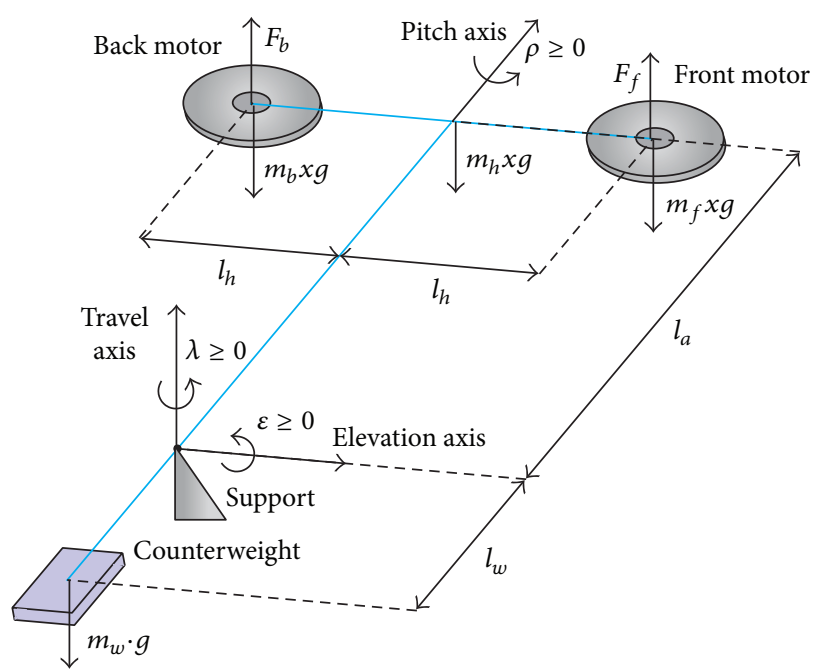

FIGURE 2: Schematic drawing of 3-DOF helicopter. Source: [10].

on which $K$ is the optimal controller associated with (19) and (20) or (21), (22), and (23).

\section{3-DOF Helicopter}

Consider the schematic model in Figure 2 of the 3-DOF helicopter [10] shown in Figure 1. This equipment is a patrimony of Control Research Laboratory at FEIS, UNESP. Two DC motors are mounted at the two ends of a rectangular frame and drive two propellers. A positive voltage applied to the front motor causes a positive pitch while a positive voltage applied to the back motor causes a negative pitch (pitch angle $(\rho))$. A positive voltage to either motor also causes an elevation of the body (elevation angle $(\varepsilon)$ of the arm). If the body pitches, the thrust vectors result in a travel of the body (travel angle $(\lambda)$ of the arm) as well. The objective of this experiment is to design a control system to track and regulate the elevation and travel of the 3-DOF helicopter [10]. 
TABLE 1: Helicopter parameters.

Power constant of the propeller (found experimentally)

Mass of the helicopter body $(\mathrm{kg})$

Mass of counterweight $(\mathrm{kg})$

Mass of the whole front of the propeller $(\mathrm{kg})$

Mass of the whole back of the propeller $(\mathrm{kg})$

Distance between each axis of pitch and motor $(\mathrm{m})$

Distance between the lift axis and helicopter (m)

Distance between elevation axis and counterweight $(\mathrm{m})$

Gravitational constant $\left(\mathrm{m} / \mathrm{s}^{2}\right)$
0.1188

1.15

1.87

$m_{h} / 2$

$m_{h} / 2$

0.1778

0.6604

0.4699

9.81
The state space model that describes the helicopter is [10]

$$
\left[\begin{array}{c}
\dot{\varepsilon} \\
\dot{\rho} \\
\dot{\lambda} \\
\ddot{\varepsilon} \\
\ddot{\rho} \\
\ddot{\lambda} \\
\dot{\xi} \\
\dot{\chi}
\end{array}\right]=A\left[\begin{array}{c}
\varepsilon \\
\rho \\
\lambda \\
\dot{\varepsilon} \\
\dot{\rho} \\
\dot{\lambda} \\
\xi \\
\chi
\end{array}\right]+B\left[\begin{array}{l}
V_{f} \\
V_{b}
\end{array}\right] .
$$

The variables $\xi$ and $\chi$ represent the integrals of the angles $\varepsilon$ of yaw and $\lambda$ of travel, respectively. Matrices $A$ and $B$ are presented in sequence:

$$
\begin{aligned}
& A=\left[\begin{array}{cccccccc}
0 & 0 & 0 & 1 & 0 & 0 & 0 & 0 \\
0 & 0 & 0 & 0 & 1 & 0 & 0 & 0 \\
0 & 0 & 0 & 0 & 0 & 1 & 0 & 0 \\
0 & 0 & 0 & 0 & 0 & 0 & 0 & 0 \\
0 & 0 & 0 & 0 & 0 & 0 & 0 & 0 \\
0 & \frac{2 m_{f} l_{a}-m_{w} l_{w} g}{2 m_{f} l_{a}^{2}+2 m_{f} l_{h}^{2}+m_{f} l_{w}^{2}} & 0 & 0 & 0 & 0 & 0 & 0 \\
1 & 0 & 0 & 0 & 0 & 0 & 0 & 0 \\
0 & 0 & 1 & 0 & 0 & 0 & 0 & 0
\end{array}\right], \\
& B=\left[\begin{array}{cc}
0 & 0 \\
0 & 0 \\
0 & 0 \\
\frac{l_{a} k_{f}}{m_{w} l_{w}^{2}+2 m_{f} l_{a}^{2}} & \frac{l_{a} k_{f}}{m_{w} l_{w}^{2}+2 m_{f} l_{a}^{2}} \\
\frac{1}{2} \frac{k_{f}}{m_{f} l_{h}} & -\frac{1}{2} \frac{k_{f}}{m_{f} l_{h}} \\
0 & 0 \\
0 & 0 \\
0 & 0
\end{array}\right] .
\end{aligned}
$$

Details of the model constants are given in Table 1. To add robustness to the system without any physical change, a 30\% drop in power of the back motor is forced by inserting a timer switch connected to an amplifier with a gain of 0.7 in tension acting directly on engine, and thus a polytope of two vertexes is constituted with an uncertainty in the input matrix of the system acting on the helicopter voltage between $0.7 V_{b}$ and $V_{b}$. The polytope is described as follows.
Vertex 1 (100\% of the amplifier gain):

$$
\begin{aligned}
& A_{1}=\left[\begin{array}{cccccccc}
0 & 0 & 0 & 1 & 0 & 0 & 0 & 0 \\
0 & 0 & 0 & 0 & 1 & 0 & 0 & 0 \\
0 & 0 & 0 & 0 & 0 & 1 & 0 & 0 \\
0 & 0 & 0 & 0 & 0 & 0 & 0 & 0 \\
0 & 0 & 0 & 0 & 0 & 0 & 0 & 0 \\
0 & -1.2304 & 0 & 0 & 0 & 0 & 0 & 0 \\
1 & 0 & 0 & 0 & 0 & 0 & 0 & 0 \\
0 & 0 & 1 & 0 & 0 & 0 & 0 & 0
\end{array}\right], \\
& B_{1}=\left[\begin{array}{ccc}
0 & 0 & \\
0 & 0 \\
0 & 0 & \\
0.0858 & 0.0858 \\
0.5810 & -0.5810 \\
0 & 0 & \\
0 & 0 &
\end{array}\right] .
\end{aligned}
$$

Vertex 2 (70\% of the amplifier gain):

$$
\begin{aligned}
& A_{2}=\left[\begin{array}{cccccccc}
0 & 0 & 0 & 1 & 0 & 0 & 0 & 0 \\
0 & 0 & 0 & 0 & 1 & 0 & 0 & 0 \\
0 & 0 & 0 & 0 & 0 & 1 & 0 & 0 \\
0 & 0 & 0 & 0 & 0 & 0 & 0 & 0 \\
0 & 0 & 0 & 0 & 0 & 0 & 0 & 0 \\
0 & -1.2304 & 0 & 0 & 0 & 0 & 0 & 0 \\
1 & 0 & 0 & 0 & 0 & 0 & 0 & 0 \\
0 & 0 & 1 & 0 & 0 & 0 & 0 & 0
\end{array}\right], \\
& B_{2}=\left[\begin{array}{ccc}
0 & 0 & \\
0 & 0 & \\
0 & 0 & \\
0.0858 & 0.0601 \\
0.5810 & -0.4067 \\
0 & 0 & \\
0 & 0 &
\end{array}\right] .
\end{aligned}
$$

\section{Comparison of Techniques for Controller $K$ Design and Optimization}

To verify the efficiency of the new optimization technique practical applications of the controllers were carried out, in order to view the controllers acting in real systems subject to failures. 


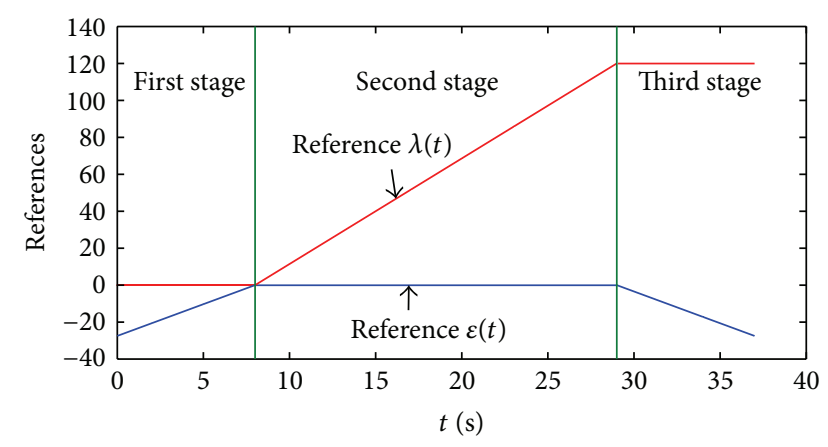

FIGURE 3: Tracking curves for $\varepsilon$ and $\lambda$.

The trajectory of the helicopter was divided into three stages as shown in Figure 3. The first stage is to elevate the helicopter $27.5^{\circ}$ reaching the yaw angle $\varepsilon=0^{\circ}$. In the second stage the helicopter travels $120^{\circ}$, keeping the same elevation; that is, the helicopter reaches $\lambda=120^{\circ}$ with reference to the launch point. In the third stage the helicopter performs the landing recovering the initial angle $\varepsilon=-27.5^{\circ}$. During the landing stage, more precisely in the instant $22 \mathrm{~s}$, the helicopter loses $30 \%$ of the power back motor. The robust controller should maintain the stability of the helicopter and have small oscillation in the occurrence of this failure.
Fixing the decay rate grater or equal to 0.8 , the following was designed: a controller with extended stability (Theorem 3) using the optimization (Theorem 4), controllers with projective stability (Theorem 5) using the optimization (Theorem 6), and the new proposed formulation for extended stability (Theorem 7) also with the improved optimization (Theorem 8) to perform the practical application. The value of the relaxation constant $a$ in the LMIs of Theorem 3 can be adequately specified by the designer. In this example we did not find difficulty in specifying $a=10^{-6}$. The designer has an alternative to perform a one-dimensional search to set this constant. Theorems 3 and 5 hypothesis establishes a sufficiently low time variation of $\alpha$. However, for comparison purposes of Theorems 3 and 5 with Theorem 7, the same abrupt loss of power test made with controller (48) was done with controllers (46) and (44).

Respectively, the controllers norm was 56.47 for the controller designed with extended stability (Theorem 3 ) and optimization (Theorem 4), 110.46 for the controller designed with projective stability (Theorem 5) and optimization (Theorem 6), and 44.84 for the controller designed with the new proposed formulation for extended stability (Theorem 7) and optimization (Theorem 8).

Below we have the controller designed with the LMIs from Theorem 3 and optimization from Theorem 4 for the application illustrated in Figure 4 and its norm:

$$
\begin{gathered}
K=\left[\begin{array}{cccccccc}
23.7152 & 12.9483 & -9.8587 & 18.7322 & 4.9737 & -14.3283 & 10.7730 & -2.6780 \\
33.8862 & -15.2923 & 11.6132 & 25.4922 & -6.0776 & 16.5503 & 15.8350 & 3.4475
\end{array}\right] \\
\|K\|=56.47 .
\end{gathered}
$$

Below we have the controller designed with the LMIs from Theorem 5 and optimization from Theorem 6 for the application illustrated in Figure 5 and its norm:

$$
K=\left[\begin{array}{cccccccc}
50.7121 & 28.7596 & -35.1829 & 29.8247 & 7.9563 & -41.0906 & 28.8974 & -11.7405 \\
66.5405 & -31.9853 & 34.7642 & 38.3173 & -9.9376 & 42.0298 & 38.3418 & 11.8207
\end{array}\right],
$$

Below we have the controller designed with the LMIs from Theorem 7 and optimization from Theorem 8 for the application illustrated in Figure 6 and its norm:

$$
K=\left[\begin{array}{cccccccc}
18.8559 & 12.5461 & -11.3752 & 13.9628 & 4.5026 & -15.1159 & 9.1565 & -3.4071 \\
27.8671 & -10.8534 & 7.8933 & 20.0978 & -4.5951 & 11.3897 & 13.6784 & 2.3623
\end{array}\right],
$$




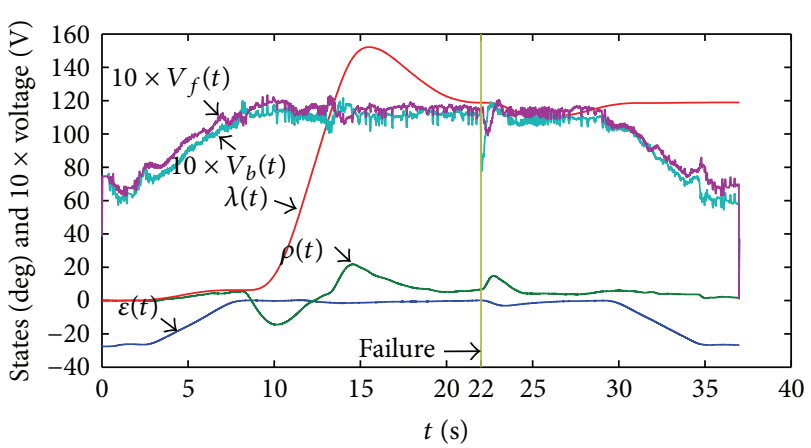

Figure 4: Practical application of the $K$ designed by extended stability (Finsler's Lemma) with the optimization method (Theorems 3 and 4). Source: [8].

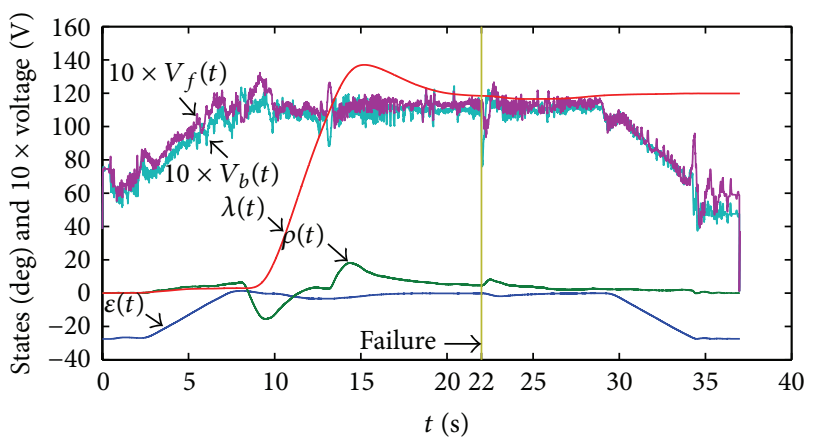

FIgURE 5: Practical application of the $K$ designed by projective stability (reciprocal projection lemma) with the optimization method (Theorems 5 and 6). Source: [8].

In Figures 4, 5, and 6 the states are shown: elevation $(\varepsilon)$, pitch $(\rho)$, and travel $(\lambda)$ in degrees for the trajectory previously stated with the application of the controllers (44), (46), and (48), respectively. All three figures also show, respectively, the control signals (voltage) in the front $\left(V_{f}\right)$ and back $\left(V_{b}\right)$ engines, in which it is possible to verify that the control signals in Figure 6 are smoother compared with those of Figures 5 and 4 . This smoothness is due to the fact that the norm of controller (48) is smaller than the others.

Note that even if the proposed method has smaller norm than the ones presented in [8], the transients before and after failure are almost the same with small differences in amplitude.

4.1. General Comparison of the Design and Optimization Methods. A generic comparison between the three methods of design and optimization was carried out to obtain more satisfactory results on what would be the best way to optimize the norm of $K$. One thousand polytopes of second order uncertain systems were randomly generated, with only one uncertain parameter (two vertexes). The one thousand polytopes were generated feasible in at least one case of design and optimization for $\gamma=0.5$ and the consequences of the $\gamma$ increase were analyzed as Figure 7 shows.

As we can see in Figure 7, the proposed formulation for extended stability with the optimization method showed

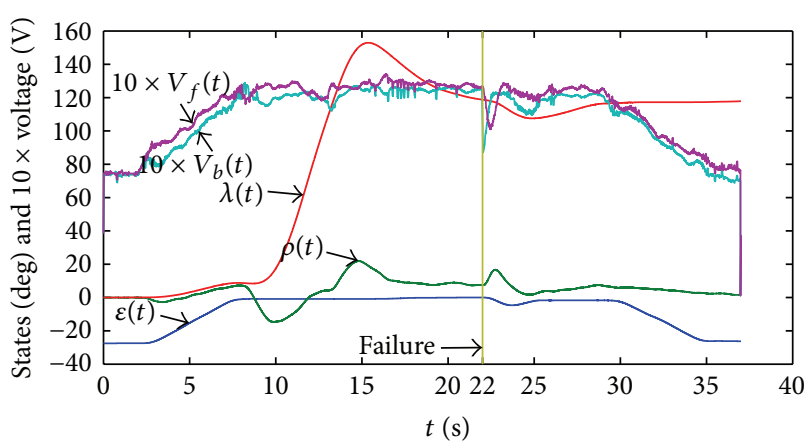

FIGURE 6: Practical application of the $K$ designed by the proposed formulation for extended stability with the optimization method (Theorems 7 and 8).

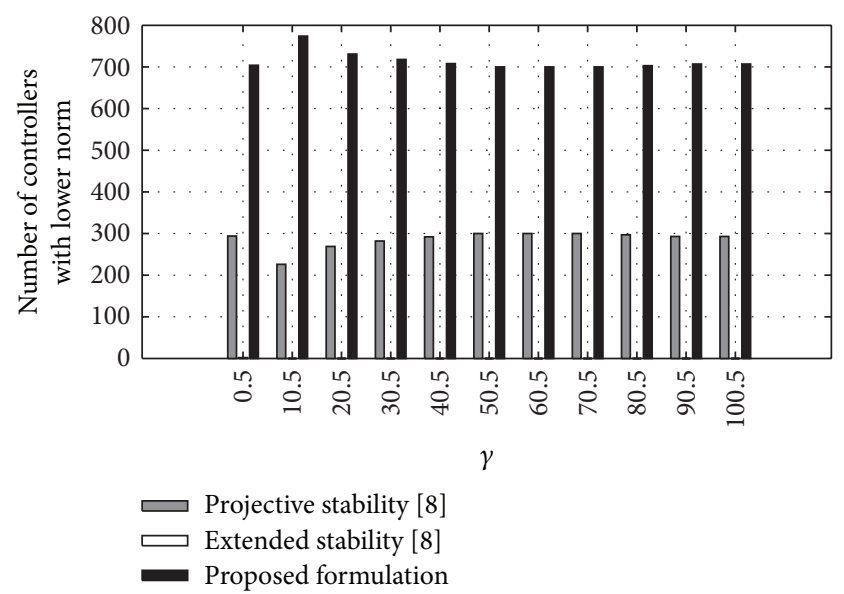

Figure 7: Number of controllers with lower norm for the design methods considering the increase in $\gamma$.

better results for the norm of the one thousand polytopes of second order uncertain systems randomly generated. This happened for all situations of $\gamma$ increase, that is, from 0.5 to 100.5 due to the insertion of the polytopic matrices $Y_{1 j}$ and $Y_{2 j}$, relaxing more the LMIs when compared to the existing formulation where there were only the polytopic Lyapunov matrices $Q_{j}$, thus allowing more efficient optimization using LMIs (31) and (32). Results for extended stability [8] are so small that they hardly appear in the bar chart due to the magnitude values of the other two methods.

\section{Conclusions}

The new proposed formulation for extended stability with the optimization method showed better results compared with those presented in [8]. Controllers were implemented for all methods in 3-DOF helicopter and for the performance of controllers in the system; it was found that the proposed method showed better results requiring lower energy expenditure. The controller designed by the proposed technique presented in this paper showed the norm reasonably smaller than the existing techniques; in addition to this for the existing techniques the abrupt variation of the uncertainty 
in the failure was made only for comparison purposes, while the new formulation allows rapid changes of $\alpha$, because the Lyapunov matrix is not polytopic, thus showing the advantage of the proposed method for application.

In the one thousand randomly generated polytopes analysis, the technique proposed in this paper proved to be better in all cases of comparison. The respective controllers design was made using MATLAB's package LMI control toolbox [5].

An immediate step in this work fits to the intelligent automatic control through the design of fuzzy control systems based on Takagi-Sugeno models.

\section{Conflict of Interests}

The authors declare that there is no conflict of interests regarding the publication of this paper.

\section{Acknowledgments}

The authors would like to thank the Brazilian agencies CAPES, CNPq, and FAPESP which have supported this research.

\section{References}

[1] S. Boyd, L. El Ghaoui, E. Feron, and V. Balakrishnan, Linear Matrix Inequalities in System and Control Theory, vol. 15 of SIAM Studies in Applied Mathematics, 2nd edition, 1994.

[2] C. Chen, Linear System Theory and Design, Oxford Series in Electrical and Computer Engineering, Oxford University Press, New York, NY, USA, 3rd edition, 1999.

[3] Q. Yang and M. Chen, "Robust control for uncertain linear system subject to input saturation," Journal of Applied Mathematics, vol. 2014, Article ID 803842, 12 pages, 2014.

[4] S. He, Z. Wu, H. Shen, Y. Yin, and Q. Zhu, "Robust control, optimization, and applications to markovian jumping systems," Abstract and Applied Analysis, vol. 2014, Article ID 582549, 3 pages, 2014.

[5] P. Gahinet, A. Nemirovski, A. J. Laub, and M. Chilali, LMI Control Toolbox-For Use with MATLAB, The Math Works Inc., 1995.

[6] R. E. Skelton, T. El Iwasaki, and K. Grigoriadis, A Unified Algebric Approach to Control Design, Taylor \& Francis, Bristol, Pa, USA, 1997.

[7] V. Solo, "On the stability of slowly time-varying linear systems," Mathematics of Control, Signals, and Systems, vol. 7, no. 4, pp. 331-350, 1994.

[8] L. F. S. Buzachero, E. Assunção, M. C. M. Teixeira, and E. R. P. da Silva, "New techniques for optimizing the norm of robust controllers of polytopic uncertain linear systems," in Frontiers in Advanced Control Systems, pp. 75-100, InTech, 2012.

[9] V. J. S. Leite and P. L. D. Peres, "An improved LMI condition for robust D-stability of uncertain polytopic systems," IEEE Transactions on Automatic Control, vol. 48, no. 3, pp. 500-504, 2003.

[10] Quanser, 3-DOF Helicopter Reference Manual, 2002.

[11] E. Assunção, M. C. Teixeira, F. A. Faria, N. A. da Silva, and R. Cardim, "Robust state-derivative feedback LMI-based designs for multivariable linear systems," International Journal of Control, vol. 80, no. 8, pp. 1260-1270, 2007.
[12] M. C. de Oliveira and R. E. Skelton, "Stability tests for constrained linear systems," in Perspectives in Robust Control, vol. 268 of Lecture Notes in Control and Information Sciences, pp. 241-257, Springer, Berlin, Germany, 2001.

[13] G. Pipeleers, B. Demeulenaere, J. Swevers, and L. Vandenberghe, "Extended LMI characterizations for stability and performance of linear systems," Systems and Control Letters, vol. 58, no. 7, pp. 510-518, 2009.

[14] V. J. S. Leite and P. L. D. Peres, "Robust control through piecewise Lyapunov functions for discrete time-varying uncertain systems," International Journal of Control, vol. 77, no. 3, pp. 230 238, 2004.

[15] W. Chang, J. B. Park, H. J. Lee, and Y. H. Joo, "LMI approach to digital redesign of linear time-invariant systems," IEE Proceedings: Control Theory and Applications, vol. 149, no. 4, pp. 297302, 2002. 

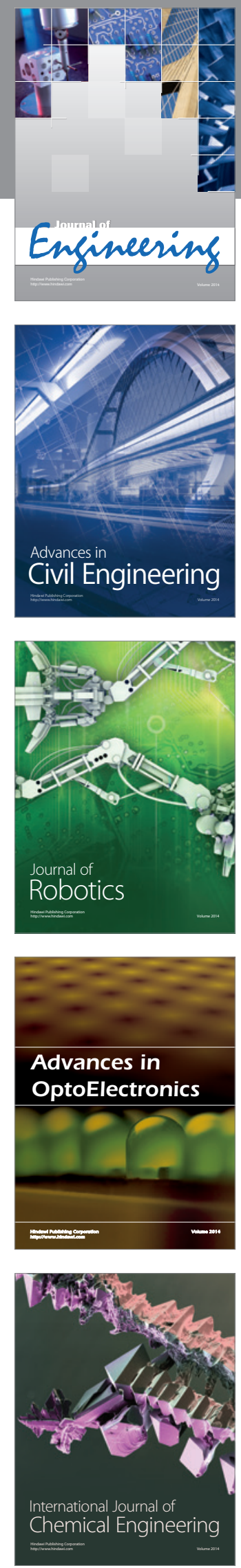

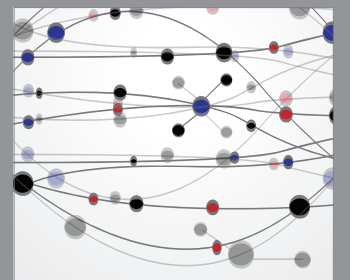

The Scientific World Journal
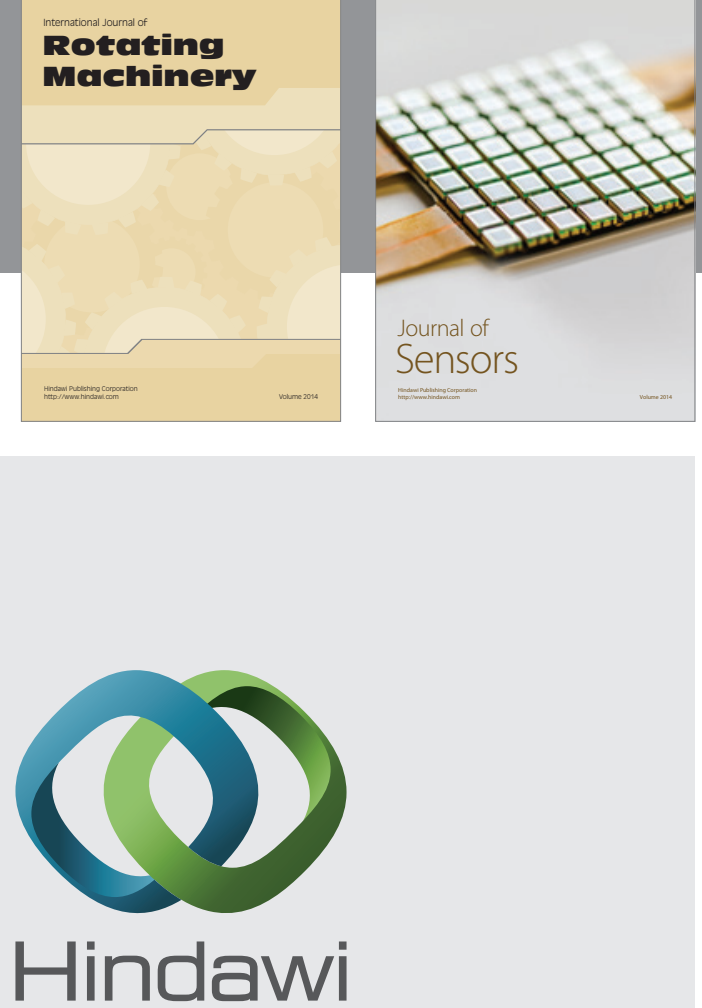

Submit your manuscripts at http://www.hindawi.com
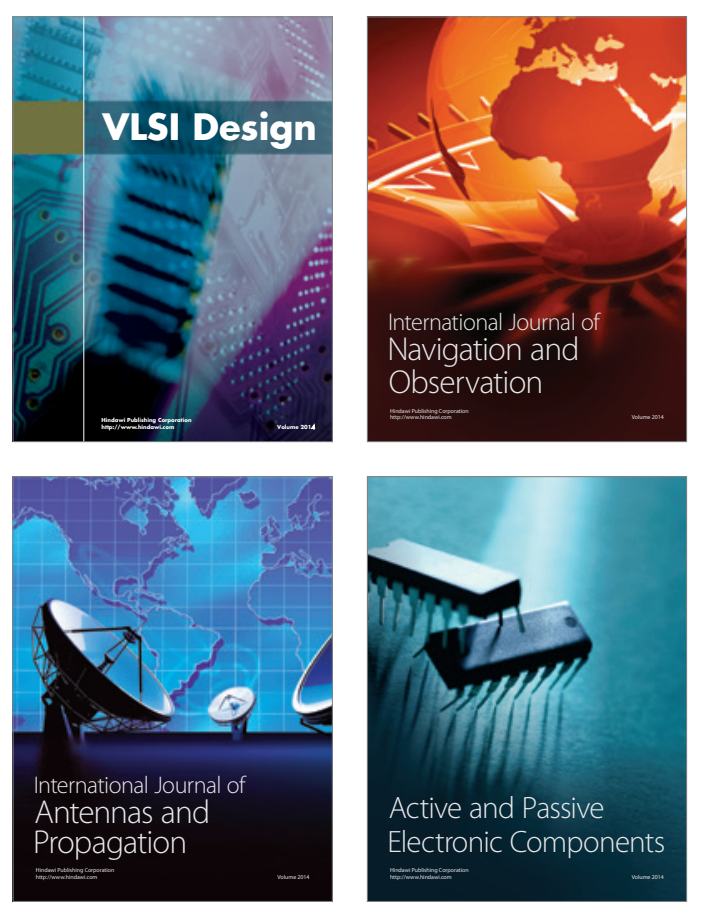
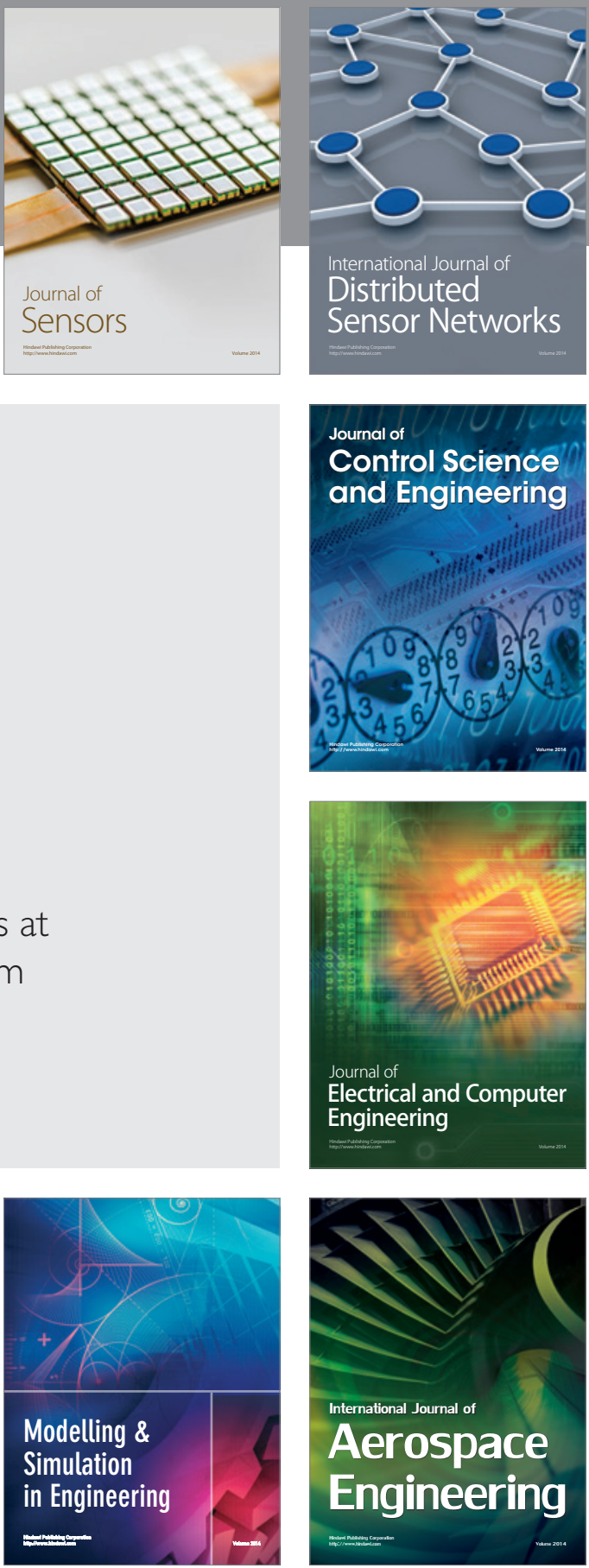

Journal of

Control Science

and Engineering
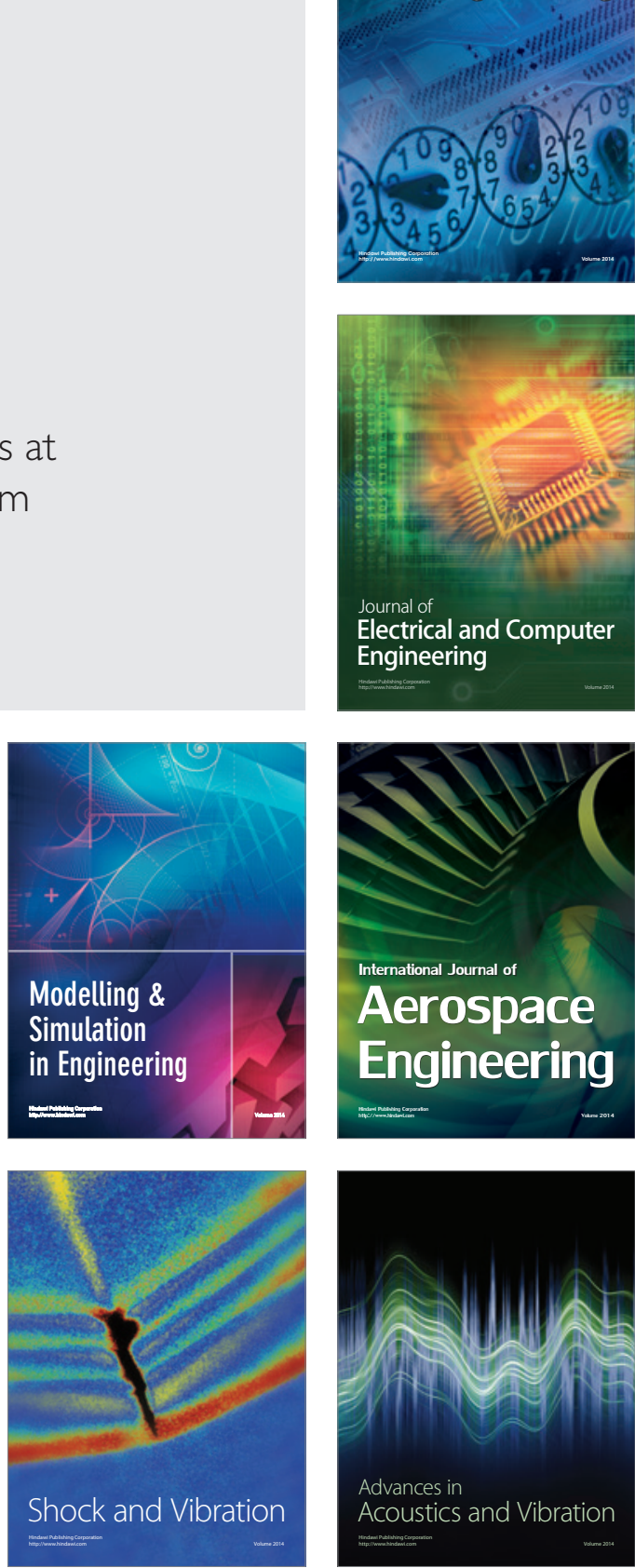